\title{
Channel Allocation for Multiple Channels Multiple Interfaces Communication in Wireless Ad Hoc Networks
}

\author{
Trung-Tuan Luong, Bu-Sung Lee, and C.K. Yeo \\ School of Computer Engineering \\ Nanyang Technological University, Nanyang Avenue, Singapore 639798 \\ $\{$ Y050089, bslee, asckyeo $\}$ @ntu.edu.sg
}

\begin{abstract}
A major drawback of multi-hop communication in ad hoc network is the bandwidth scarcity along the forwarding path of data packet. The main reason for the lack of bandwidth or the exponential reduction in the bandwidth is the contention for bandwidth between nodes along the path. In this paper we will demonstrate one way to overcome this problem through the use of multiple channels multiple interfaces (MCMI). We investigate different forwarding channel selection schemes for MCMI communication with Destination Sequenced Distance Vector protocol: same channel, random and round robin. Based on our analysis and simulation results we have shown that MCMI protocol significantly improves the channel capacity while maintaining low end-to-end delay. Forwarding channel selection policies play an important role in determining the performance of the MCMI. Among the channel assignment policies under study, round robin policy provides the best performance. The results provide a baseline for evaluating bandwidth of multiple channels multiple interfaces networks.
\end{abstract}

Keywords: Multiple channel communication, channel interference, channel assignment, bandwidth.

\section{Introduction}

Wireless communication has become an indispensable part of modern day-to-day life. Research in ad hoc networks has focused on single channel networks, in which a common channel is desired for simple routing control in multi-hop networks. A well known fact that is affecting the performance of ad hoc networks is the significant throughput degradation along the multi-hop path [1]. When a single channel is used both for incoming and outgoing traffic, throughput is halved as they use the same radio channel. That means when one node is transmitting, its neighbors nodes must all be in listening mode otherwise collision will occur. This problem is amplified across the network, and after a few hops the bandwidth is reduced significantly. To overcome the throughput degradation problem, a natural approach is to use multiple channels in which nodes work on different channels in an interference area.

A. Das et al. (Eds.): NETWORKING 2008, LNCS 4982, pp. 87 98, 2008.

(C) IFIP International Federation for Information Processing 2008 
Recent research and commercial efforts in the wireless networking industry have focused on a new class of ad hoc networks: multiple channels multiple interfaces ad hoc networks [1]. These networks are characterized by a set of fixed nodes with multiple wireless interfaces utilizing multiple orthogonal channels at the same time over a given swath of spectrum such as IEEE $802.11 \mathrm{a} / \mathrm{b} / \mathrm{g}$. Simultaneous operation of multiple channels provides the following advantages:

- Capacity enhancement: A node with two interfaces can send packets on two channels simultaneously. More importantly, forwarding nodes can both send and receive at the same time. Using appropriate protocols for channel selection and assignment, such a system can provide significantly greater capacity than a single interface system

- Load sharing: Load sharing occurs where a traffic flow is distributed among the available connections to achieve lower latency and increase robustness to network.

- Channel failure recovery: Graceful degradation and robustness against channel errors is possible by employing frequency diversity. Frequency diversity can be achieved by using multiple interfaces and operating each on different channel frequencies.

In this paper, we implement the Destination Sequenced Distance Vector (DSDV) for multiple channels multiple interfaces wireless ad hoc networks (DSDV-MCMI) to reduce the bandwidth degradation problem. In DSDV-MCMI, we investigated three different policies for forwarding channel selection: same channel, random channel and round robin channel to evaluate the channel interference along the multi-hop path. Our simulation results show that DSDVMCMI protocol significantly improves the network goodput while maintaining low end-to-end delay. Among the three different channels selection policies for MCMI, round robin policy, where the packets are forwarded based on the packet incoming channel, is the best at preventing channel interference; which results in shortest end-to-end delay time.

Our paper outline is as follows. Section 2 briefly discusses the related work. In Section 3, we present the multiple channels multiple interfaces protocol with DSDV-MCMI. The simulation and results analysis are given in Section 4. Finally, Section 5 concludes the paper.

\section{Related Work}

We can categorize strategies for using multiple channels into two groups depending on the number of interfaces used: single interface and multiple interfaces. The first strategy is to enable a single wireless interface card to access multiple channels [23]. For example, So and Vaidya 3] propose a scheme that allows wireless devices to communicate on multiple channels using a single interface card. The scheme requires frequent channel switching, which incurs considerable overhead on the current hardware. The other strategy is to use multiple interface cards; Nasipuri et al. 456] propose a class of multi-channel carrier sense multiple access 
MAC protocols where all nodes have an interface on each channel. These proposed protocols use different metrics to choose the channel for communication between nodes. The metric may simply use the idle channel [4, or the signal power observed at the sender [5], or the received signal power at the receiver [6]. Wu et al [7] propose a MAC layer solution that requires two interfaces. One interface is assigned to common channel for control purposes, and the second interface is switched between the remaining available channels for transmitting data. Lee 8] proposed a class of proactive routing protocol for multi channels, which uses one control channel and $\mathrm{N}$ data channels. The nodes exchange control packages on the control channel to negotiate the best channel for the receiver's data channel.

Most past research in the use of multiple channels require either modification of the MAC layer protocol or changes to IEEE 802.11. Compared to previous research, our proposed protocol focus on exploit the multiple channels by using concurrent multiple interfaces. The protocol does not require channel synchronization among nodes. Although there is some additional control overhead, the simulation results show that the proposed protocol significantly increase network goodput while decreasing end-to-end delay efficiently.

\section{Multiple Channels Multiple Interfaces Routing with DSDV}

\subsection{Model Description}

Module based node for ns-2. Wireless nodes are becoming multimodal. They are equipped with multiple interfaces possibly using different technologies, including wired access, and may use them concurrently. Paquereau [9] designed module based wireless node (MW-Node), a more flexible and better integrated wireless and mobile support in ns-2[10. It is not a new implementation but primarily a reorganization of already existing components. The MW-Node enables new features to be supported by the simulator. For instance, true support for multiple wireless interfaces on a single node, each may have its own routing protocol.

DSDV-MCMI. Based on the MW-Node model, we developed destination sequenced distance vector for multiple channels multiple interfaces, DSDV-MCMI, to demonstrate the multiple channels multiple interfaces routing scheme. Nodes can have more than one network interfaces as shown in Fig. 1, These interfaces are assigned to orthogonal channels so that they will not interfere with each other. Every interface has one DSDV [11] routing agent for generating control messages and forwarding data packets.

Destination-Sequenced Distance-Vector Routing (DSDV) is a table-driven routing protocol based on the Bellman-Ford algorithm. Each mobile node maintains a route to every other node in a routing table. Route entry contains destination address, next hop address and number of host to reach the destination. In order to prevent routing loop, each entry is marked with a sequence number assigned by the destination node. The sequence numbers enable node to 


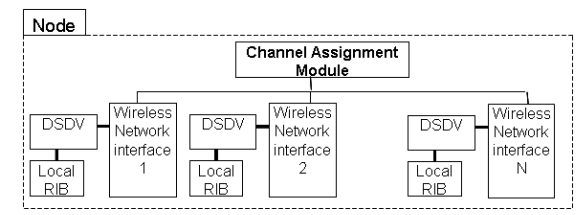

Fig. 1. Multi-interface node

distinguish stale routes from new ones. The DSDV routing agent at each interface maintains its' own routing table for all available destinations. To maintain the consistency of routing tables in a dynamically varying topology, each node's interface periodically transmits full updates or immediately transmits incremental updates when significant new information is available, such as a topology changes. We design a channel assignment module on top of the network interfaces. This module is in charge of selecting the network interface for forwarding each data packets.

\subsection{Channel Assignment Policy}

The purpose of channel assignment is to determine the forwarding channel for packet to minimize interference with other nodes. We apply the static approach 12] that assigning a common set of channels for node's interfaces. Every node has $\mathrm{N}$ interfaces, which are assigned to the same set of $\mathrm{N}$ orthogonal channels. At the source node, data packet is sent out on an interface which is randomly selected. When an intermediate node along the path receives a data packet on incoming channel $\mathrm{Ch}_{\text {in }}$; the node uses one of the following three policies to select the outgoing channel $\mathrm{Ch}_{\text {out }}$ for relaying data packet:

- Policy 1 Same channel: node will relay the packet on the same channel as the packet was received. This approach incurs high channel interference in multi-hop route, which results in long delay and packet drops that degrade the performance significantly.

$$
\mathbf{C h}_{\text {out }}=\mathbf{C h}_{\text {in }}
$$

- Policy 2 Random channel: node relays data packet on a randomly selected channel. The probability that the forwarding channel is the same as the received channel is $\frac{1}{N}$. When a node has more interfaces, the less chance that the forwarding channel is the same as the received channel.

$$
\mathrm{Ch}_{\text {out }}=\operatorname{Random}\left(\mathrm{Ch}_{0}, \mathrm{Ch}_{1}, \ldots, \mathrm{Ch}_{N-1}\right)
$$

- Policy 3 Round robin channel: when a node receive data packet need to be forwarded on $\mathrm{i}^{\text {th }}$ channel it will forward that packet on the $(\mathrm{i}+1)^{t h}$ channel.

$$
\mathrm{Ch}_{\text {out }}=\left(\mathbf{C h}_{\text {in }}+\mathbf{1}\right) \bmod (\mathrm{N})
$$




\subsection{Channel Interference}

Assuming that nodes are ideally placed along the route with hop distance 'd' as in Fig 2, all the node has transmission range ' $\mathrm{T}$ ' and carrier sensing range 'C'. The ideal placement of a node is

$$
d \leq T<2 * d
$$

The transmissions of a node interfere with transmissions of other nodes within its' sensing range. Thus sensing range is also called as interference range. The interference distance in term of number of hops along a path is:

$$
\mathrm{M}=\left\lfloor\frac{C}{d}\right\rfloor \text { hops where }\left\lfloor\frac{C}{d}\right\rfloor \text { is floor of } \frac{C}{d}
$$

This means that transmission of a node may interfere with transmission of the next $\mathrm{M}$ nodes and transmission of the previous $\mathrm{M}$ nodes along the path. We will prove the throughput by using multiple channels for transmission; the minimum number of channels required to eliminate the channel interference among forwarding nodes along the path is $(M+2)$. As shown in Fig. 2. nodes $A_{1}, A_{2}, \ldots$,

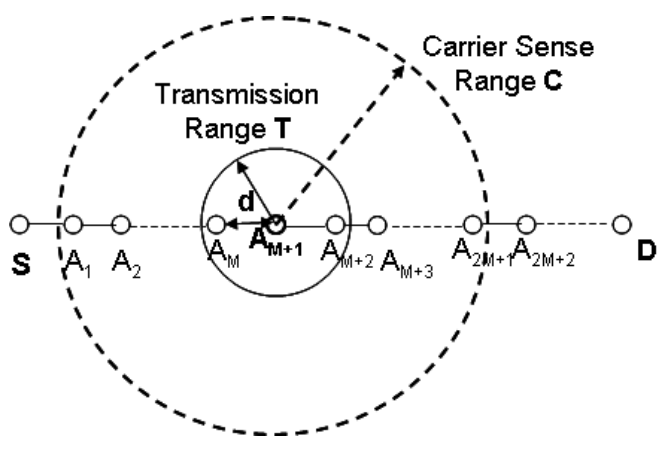

Fig. 2. Multi-hop path

$A_{M+1}$ are within interference range of each other, because the distance between $\mathrm{A}_{1}$ and $\mathrm{A}_{M+1}$ is $\mathrm{M}$ hops. Therefore the $(\mathrm{M}+1)$ data transmissions from $\mathrm{A}_{i}$ to $\mathrm{A}_{i+1}(\mathrm{i}=1,2, . ., \mathrm{M}+1)$ can interfere with each other. Due to the exposed node problem, data transmission from node $\mathrm{A}_{M+1}$ to node $\mathrm{A}_{M+2}$ can also interfere with CTS/ACK packet sent from node $\mathrm{A}_{1}$ to node $\mathrm{S}$. Thus we can see that there are $(\mathrm{M}+2)$ data transmissions interfering with each other. Hence we need at least $(\mathrm{M}+2)$ channels so that every data transmission can work on different channel to ensure these communications will not interfere with each other.

In Fig. 3. we apply policy 3 for forwarding channel selection among the set of $(\mathrm{M}+2)$ channels $\left\{\mathrm{Ch}_{0}, \mathrm{Ch}_{1}, . ., \mathrm{Ch}_{M+1}\right\}$. We can see that two transmissions use the same channel, such as link $\mathrm{S}-\mathrm{A}_{1}$ and link $\mathrm{A}_{M+2}-\mathrm{A}_{M+3}$, are $(\mathrm{M}+1)$ hops apart along the path, hence they will not interfere with each other. Thus with $(\mathrm{M}+2)$ available channels, we can use policy 3 for channel assignment for data transmission so that we can eliminate channel interference 


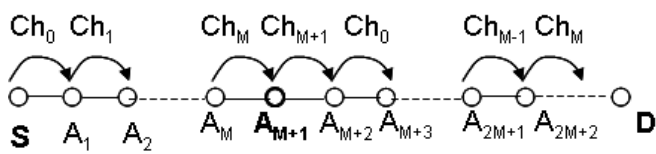

Fig. 3. Channel assignment for communication link in multi-hop path

\subsection{Capacity of a Chain of Multiple Channels Multiple Interfaces Nodes}

In this subsection, we evaluate the capacity of a single chain of nodes where packets are originated at first node and forwarded along the chain to the last node. We investigate chain of nodes under saturated condition that every node along the chain always has packet to forward. When node A select channel to forward packet, we denote $\mathrm{P}_{I}(\mathrm{~A})$ the probability that the selected channel will be interfered. Let $\mathrm{S}$ be the set of $\mathrm{N}$ available channels; $\mathrm{B}$ is channel bandwidth; $\mathrm{B}(\mathrm{A})$ is bandwidth experienced by node $\mathrm{A}$. $\mathrm{B}_{\text {Chain }}$ is the maximum bandwidth can be achieved for transmission from source to destination of the chain. $\mathrm{M}$ is the interference distance in term of number of hops and $\mathrm{L}$ is hops length of the chain.

Along the chain, we can see that the middle node such as node $\mathrm{A}_{M+1}$ in Fig. 2 is the bottleneck node, because its' data transmission may share the same channel with most number of other transmissions. Bandwidth of chain is reduced significantly because of those nodes. Thus we will evaluate the bandwidth can be experienced by the middle node $\mathrm{A}$ in the chain.

Policy 1. we have $\mathrm{Ch}_{\text {out }}=\mathrm{Ch}_{\text {in }}$, therefore two consecutive data transmissions along the path always interfere with each other. Thus channel interference always happens, $\mathrm{P}_{I}(\mathrm{~A})=1$.

If the chain is long enough, which is equal or more than $\left(2^{*} \mathrm{M}+2\right)$ hops long. We examine the middle node $\mathrm{A}_{M+1}$ as in Fig. 2] $\mathrm{A}_{M+1}$ will share the same channel with the next $M$ nodes $\mathrm{A}_{M+2}, . ., \mathrm{A}_{2 M+1}$ and the previous $\mathrm{M}$ nodes $\mathrm{A}_{1}, .$. , $\mathrm{A}_{M}$ along the path. However due to the exposed node problem, transmission from node $\mathrm{A}_{M+1}$ to node $\mathrm{A}_{M+2}$ will also interfere with CTS/ACK packet sent from node $\mathrm{A}_{1}$ to node $\mathrm{S}$. Therefore we can say that node $\mathrm{A}_{M+1}$ shares the same channel with $(2 * \mathrm{M}+1)$ other node.; this makes $\mathrm{B}\left(\mathrm{A}_{M+1}\right)=\frac{B}{2 * M+2}$. Thus the chain of nodes can achieve a maximum bandwidth of $\frac{B}{2 * M+2}$.

If hops length $\mathrm{L}<\left(2^{*} \mathrm{M}+2\right)$; the middle node will have carrier sensing range cover the whole chain, thus its' data transmission will share the same channel with all the other (L-1) transmissions along the path. So its' experienced bandwidth will be $\frac{B}{L}$. Therefore the chain can achieve a maximum bandwidth of $\frac{B}{L}$.

$$
B_{\text {Chain }}= \begin{cases}\frac{B}{2 * M+2} & \text { if } L \geq(2 * M+2) \\ \frac{B}{L} & \text { if } L<(2 * M+2)\end{cases}
$$

Policy 2. we denote $\mathrm{P}_{N I}(\mathrm{~A})$ the probability that the selected channel of A will not be interfered. $\mathrm{P}_{N I}(\mathrm{~A})=1-\mathrm{P}_{I}(\mathrm{~A})$. Let $\mathrm{K}$ be number of forwarding nodes 
along the chain excluding A stay inside carrier sensing range of A. As discussed in policy $1, \mathrm{~K}$ is $\left(2^{*} \mathrm{M}+1\right)$ if $\mathrm{L} \geq\left(2^{*} \mathrm{M}+2\right)$; otherwise $\mathrm{K}$ is $(\mathrm{L}-1)$. We can see that $\mathrm{P}_{N I}(\mathrm{~A})$ equals to the probability that node $\mathrm{A}$ selects channel $\mathrm{Ch}_{i}$ and the other K nodes select among the rest (N-1) channels $\left(\mathrm{S} \backslash\left\{\mathrm{Ch}_{i}\right\}\right)$ multiply by $\mathrm{N}$.

$$
\begin{gathered}
\mathrm{P}_{N I}(\mathrm{~A})=\frac{1}{N} *\left(\frac{N-1}{N}\right)^{K} * \mathrm{~N}=\left(\frac{N-1}{N}\right)^{K} \\
\mathrm{P}_{I}(\mathrm{~A})=1-\mathrm{P}_{N I}(\mathrm{~A})=1-\left(\frac{N-1}{N}\right)^{K}
\end{gathered}
$$

we denote $\mathrm{P}_{I}(\mathrm{~A}, \mathrm{~J})$ the probability that the selected channel of $\mathrm{A}$ will be shared with other $\mathrm{J}$ nodes

$$
\begin{gathered}
\mathrm{P}_{I}(\mathrm{~A}, \mathrm{~J})=\left(\frac{1}{N}\right)^{J+1} *\left(\frac{N-1}{N}\right)^{K-J} * N=\left(\frac{1}{N}\right)^{J} *\left(\frac{N-1}{N}\right)^{K-J} \text { where J=0.. } \\
\mathrm{B}(\mathrm{A})=\sum_{J=0}^{K}\left[P_{I}(A, J) * \frac{B}{J+1}\right]=B *\left(\frac{1}{N}\right)^{K} \sum_{J=0}^{K}\left[\frac{(N-1)^{K-J}}{J+1}\right] \\
B_{\text {Chain }}=B *\left(\frac{1}{N}\right)^{K} \sum_{J=0}^{K}\left[\frac{(N-1)^{K-J}}{J+1}\right] \text { where } K=\left\{\begin{array}{lr}
(2 * M+1) & \text { if } L \geq(2 * M+2) \\
L-1 & \text { if } L<(2 * M+2)
\end{array}\right.
\end{gathered}
$$

Policy 3. We first evaluate the long chain of nodes that $\mathrm{L} \geq\left(2^{*} \mathrm{M}+2\right)$. If $\mathrm{N}$ $\geq(\mathrm{M}+2)$, policy 3 can help to eliminate channel interference; thus $\mathrm{P}_{I}(\mathrm{~A})=0$ and $\mathrm{B}(\mathrm{A})=\mathrm{B}$. Else if $\mathrm{N}<(\mathrm{M}+2), \mathrm{P}_{I}(\mathrm{~A})=1$ and node $\mathrm{A}$ will share the same channel with next $\left\lfloor\frac{M}{N}\right\rfloor$ nodes and previous $\left\lfloor\frac{M}{N}\right\rfloor$ nodes along the chain. Node A may share the same channel with the exposed node if $\left\lfloor\frac{M+1}{N}\right\rfloor=\frac{M+1}{N}$. Therefore $\mathrm{B}(\mathrm{A})=\frac{B}{\left[\frac{M}{N}\right]+\left[\frac{M+1}{N}\right\rfloor+1}$.

Then we evaluate the short chain that $\mathrm{L}<(2 * \mathrm{M}+2)$. If $\mathrm{L} \leq \mathrm{N}$ then all the transmissions use different channel thus $\mathrm{P}_{I}(\mathrm{~A})=0$ and $\mathrm{B}(\mathrm{A})=\mathrm{B}$. Else if $\mathrm{L}>$ $\mathrm{N}$, data transmission of node $\mathrm{A}$ will share the same channel with other $\left\lceil\frac{L}{N}\right\rceil-1$ transmission. Therefore $\mathrm{P}_{I}(\mathrm{~A})=1$ and $\mathrm{B}(\mathrm{A})=\frac{B}{\left\lceil\frac{L}{N}\right\rceil}$ where $\left\lceil\frac{L}{N}\right\rceil$ is ceiling of $\frac{L}{N}$

$$
B_{\text {Chain }}= \begin{cases}B & \text { if } L \geq(2 * M+2) \operatorname{and} N \geq(M+2) \\ \frac{B}{\left\lfloor\frac{M}{N}\right\rfloor+\left\lfloor\frac{M+1}{N}\right\rfloor+1} & \text { if } L \geq(2 * M+2) \operatorname{and} N<(M+2) \\ \frac{B}{\left\lceil\frac{L}{N}\right\rceil} & \text { if } N<L<(2 * M+2) \\ B & \text { if } L \leq N\end{cases}
$$

\section{Simulation and Results}

\subsection{Verification of Chain Results}

First we simulate MCMI in a chain of nodes setup, where nodes are placed ideally $150 \mathrm{~m}$ apart from each other. The transmission range is $250 \mathrm{~m}$ and carrier sensing range is $550 \mathrm{~m}$. This is the worst-case placement, where node is placed at just above half of transmission range of each other. In this scenario, interference distance $\mathrm{M}$ is 3 hops. Packet is originated at source node with the rate of $2 \mathrm{Mbps}$, which equals to channel bandwidth; so that data transmission of the chain is 
Table 1. Bandwidth drop rate when chain's length increases

\begin{tabular}{|c|c|c|c|c|c|c|c|c|c|c|}
\hline Chain length (hops) & 1 & 2 & 3 & 4 & 5 & 6 & 7 & 8 & 9 & 10 \\
\hline $1 \mathrm{Int}$ & 1 & $\frac{1}{2}$ & $\frac{1}{3}$ & $\frac{1}{4}$ & $\overline{5}$ & $\overline{6}$ & $\frac{1}{7}$ & $\frac{1}{8}$ & $\frac{1}{8}$ & $\frac{1}{8}$ \\
\hline 2 Interface with Policy 3 & 1 & ${ }_{1}^{2}$ & $\begin{array}{l}3 \\
\frac{1}{2}\end{array}$ & $\frac{4}{2}$ & $\frac{5}{3}$ & 6 & & & $\overline{4}$ & $\overline{4}$ \\
\hline 3 Interface with Policy 3 & 1 & 1 & ${ }_{1}^{2}$ & $\frac{2}{2}$ & $\frac{3}{2}$ & $\frac{3}{2}$ & $\frac{4}{3}$ & $\frac{4}{3}$ & $\begin{array}{l}\frac{4}{3} \\
\end{array}$ & $\begin{array}{l}4 \\
\underline{1}\end{array}$ \\
\hline 4 Interface with Policy 3 & 1 & 1 & 1 & 1 & $\frac{1}{2}$ & $\frac{1}{2}$ & $\frac{1}{2}$ & $\frac{1}{2}$ & $\frac{3}{2}$ & \\
\hline 5 Interface with Policy 2 & 1 & 0.9 & 0.73 & 0.59 & 0.47 & 70.38 & 30.30 & 0.24 & $4 \stackrel{2}{0.24}$ & $4 \stackrel{2}{0} .24$ \\
\hline 5 Interface with Policy 3 & 1 & 1 & 1 & 1 & 1 & 1 & 1 & 1 & 1 & 1 \\
\hline
\end{tabular}

under saturated condition. We will measure the bandwidth can be achieved when the chain is 1-hop length and measure how the bandwidth drops when chain is longer. We increases numbers of interfaces equipped by nodes and apply different channel selection policy to verify our analysis.

We calculate the drop rate of maximum bandwidth achieved of chain of $\mathrm{N}$ hops length compared to 1-hop length chain according to equation (2), (3), (4). The result is summarized in Table 1 Fig. 4 shows the comparison results of our

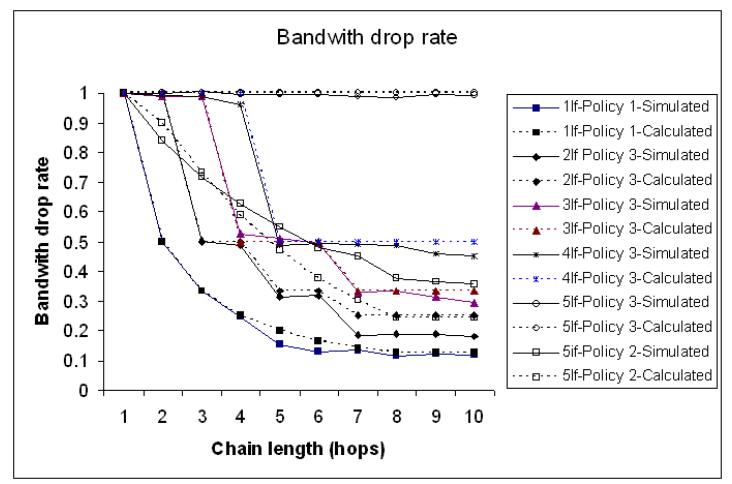

Fig. 4. Bandwidth drop rate when number of hops increases

simulation and analysis. We can see that our simulation's results are very close to the analysis results shown in Table 1. When the chain gets longer, packet must traverse more hops. Packets' end-to-end delay increases while bandwidth decreases.

\subsection{Simulation of MCMI in Two Dimensions Multihops Network Environment}

We evaluated the performance of MCMI in static ad hoc network of map size $1200 \mathrm{mx} 800 \mathrm{~m}$ with network node density of 80 nodes. This setting helps to ensure that network is crowded enough so that we can have a path between any nodes; and network is also scarce enough so that the path length is long for evaluating effects of different channel allocation policies. The average hop counts of our 
Table 2. Simulation Setting

\begin{tabular}{ll}
\hline Parameter & Setting \\
\hline Traffic model & 20CBR connections \\
Packet size & 512 bytes \\
Packet sending rate & $2 \mathrm{pkt} / \mathrm{s}-12 \mathrm{pkts} / \mathrm{s}$ \\
Mac & $802.112 \mathrm{Mbps}$ \\
Transmission range & $250 \mathrm{~m}$ \\
Carrier sensing range & $550 \mathrm{~m}$ \\
Map size & $1200 \mathrm{mx} 800 \mathrm{~m}$ \\
Node number & 80 \\
Simulation time & $300 \mathrm{~s}$ \\
\hline
\end{tabular}

simulation are 3.5 hops. We simulate $20 \mathrm{CBR}$ connections with 512 bytes packet size in 300 seconds. The packet rate increases from 2 packets/s to 12 packets/s to vary the traffic load. A summary of the simulation setting is given as shown in Table 2. The following performance metrics are used to evaluate the simulation results:

- Goodput: the number of useful bits per unit of time forwarded by the network from a certain source address to a certain destination, excluding protocol overhead, and excluding retransmitted data packets

- End-to-end delay: the average time it takes for a packet to traverse the network from its source to destination.

\subsection{Result Analysis}

\section{Goodput}

Experiment 1: Effects of number of Interfaces, N. Fig. [5] shows the goodput results at different traffic load for different number of channels. The goodput performance of $\mathrm{N}$-interfaces is normalized against the performance of 1-interface. With increasing number of node's interface, nodes can receive packet on one interface and send packet on other interface at the same time. Thus with more interfaces, node can share the traffic load better, which help to reduce the congestion. Therefore as expected when number of interfaces increases, the goodput increases. We do not expect the goodput to increase linearly as the number of interfaces increases, because communication paths can crossover and interfere each others. This causes bandwidth reduction in all the crossover communication path.

Experiment 2: Effects of Forwarding Channel Assignment Policy. The normalized goodput performances of three channel assignment policies is compared against policy 1 in three-interface network are shown in Fig. 6. Policy 3 is the best as it diversifies channel usages, which results in greatest goodput achieved. Policy 1 does nothing to prevent channel interference, thus its' goopdut performance is much less than that of policy 3. Policy 2 does a little effort to prevent 


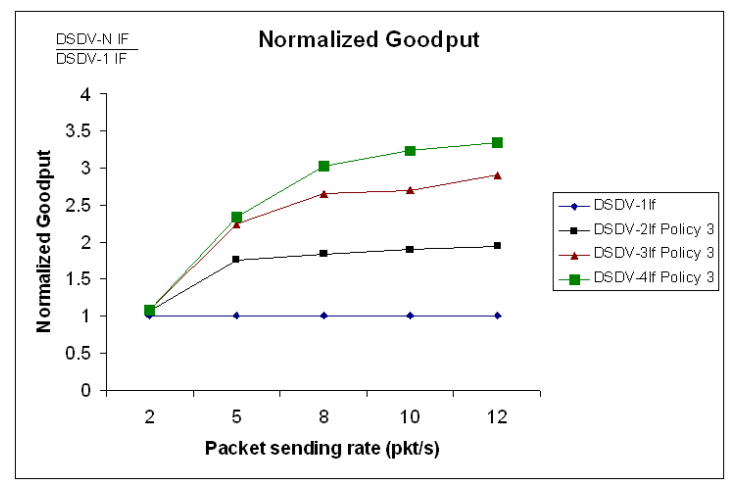

Fig. 5. Normalized goodput regarding number of interfaces

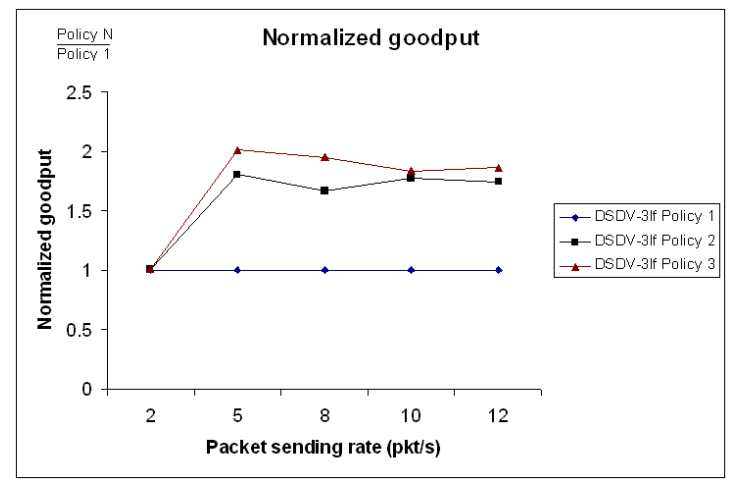

Fig. 6. Normalized goodput regarding forwarding channel selection policy

channel interference by selecting forwarding channel randomly, which still has probability of channel interference. Thus policy 2 performs slightly worse than policy 3 .

\section{End-to-End Delay}

Experiment 1: Effects of number of Interfaces, $N$. Fig. 7 shows the corresponding results of end-to-end delay for different number of network interface used for transmission, as the traffic load is increased. A key observation is that, in a congested network, packets will be kept in queue for very long, waiting for channel availability; which resulted in either long end to end delay or packet dropped. When node has more interfaces, it will be able to forward packets through all the interfaces. Thus packets need not wait long in queue; as the result, with more interface the end-to-end delay decreases considerably. For example at packet sending rate of $10 \mathrm{pkt} / \mathrm{s}$, the end to end delay can be reduced by 2, 4, 6 times that of a single interface when node has 2,3 and 4 interfaces respectively. 


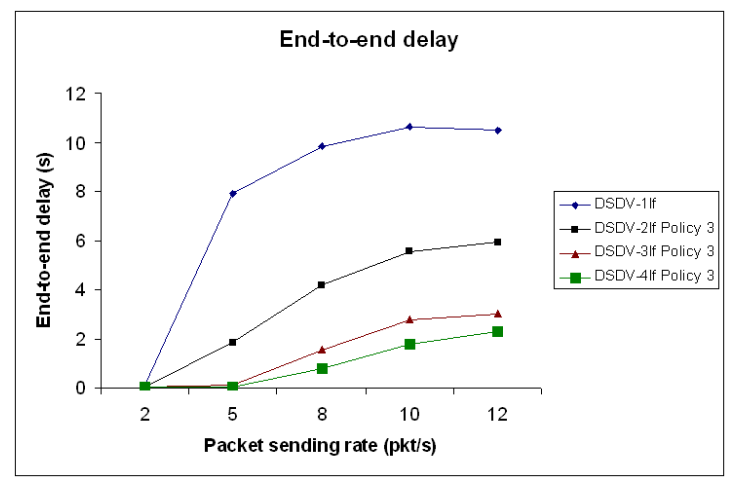

Fig. 7. End-to-end delay regarding number of interfaces

Experiment 2: Effects of Forwarding Channel Assignment Policy. Fig. 8 presents the end to end delay performance of three channel assignment policies in three-interface node configuration with varying traffic load. Forwarding data packets on the same channel as the receiving channel incurs the greatest channel interference, thus policy 1 has the highest end-to-end delay. Policy 3 is the best as it diversifies channel usages, thus it can help reduce channel interference, which results in shortest end-to-end delay. The performance of policy 2 is slightly worse than policy 3 .

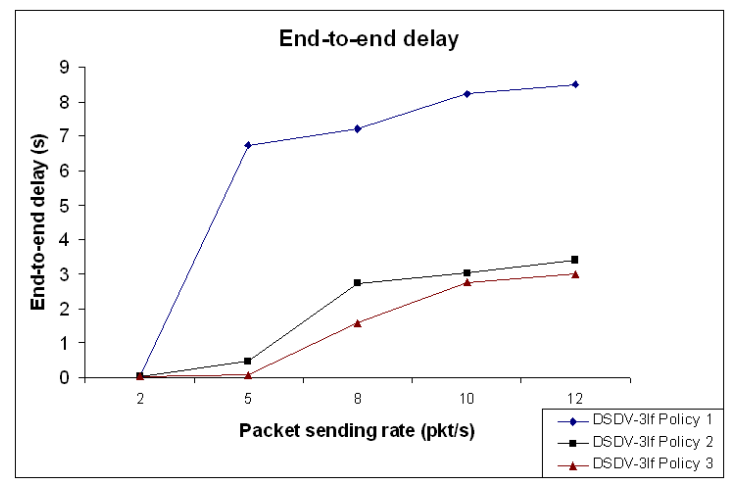

Fig. 8. End-to-end delay regarding forwarding channel selection policy

\section{Conclusion}

We presented a multiple channels multiple interfaces ad hoc network routing protocol, DSDV-MCMI, which utilizes multiple channels to enable simultaneous multiple transmission to improve network capacity. This proposed scheme does not require modification to the current IEEE 802.11 MAC protocol. Simulation 
results shows that DSDV-MCMI exploits multiple channels multiple interfaces to improve network capacity and reduce channel interference. With more equipped network interfaces, we can achieve higher goodput and shorter end-to-end delay time. We also suggested some policies for forwarding channel selection: same channel, random channel and round robin channel; these policies play an important role in determining performance of MCMI. However, there is also an issue of crossover interference among the communication path and one needs a deeper understanding before a satisfactory solution is found.

\section{References}

1. Bahl, P., Adya, A., Padhye, J., Walman, A.: Reconsidering wireless systems with multiple radios. SIGCOMM Comput. Commun. Rev. 34(5), 39-46 (2004)

2. Chandra, R., Bahl, P., et al.: Multinet: Connecting to multiple ieee 802.11 networks using a single wireless card. In: Proceedings of IEEE Infocom 2004, Hongkong (March 2004)

3. So, J., Vaidya, N.H.: Multi-channel mac for ad hoc networks: handling multichannel hidden terminals using a single transceiver. In: MobiHoc 2004: Proceedings of the 5th ACM international symposium on Mobile ad hoc networking and computing, pp. 222-233. ACM Press, New York (2004)

4. Nasipuri, A., Zhuang, J., Das, S.R.: A multichannel csma mac protocol for multihop wireless networks. In: Wireless Communications and Networking Conference. WCNC, vol. 3, pp. 1402-1406. IEEE, Los Alamitos (1999)

5. Nasipuri, A., Das, S.: Multichannel csma with signal power-based channel selection for multihop wireless networks. In: Vehicular Technology Conference, 2000. IEEE VTS-Fall VTC 2000. 52nd, vol. 1(1), pp. 211-218 (2000)

6. Jain, N., Das, S., Nasipuri, A.: A multichannel csma mac protocol with receiverbased channel selection for multihop wireless networks. In: Proc. IEEE IC3N, Phoenix (October 2001)

7. Wu, S.-L., Lin, C.-Y., Tseng, Y.-C., Sheu, J.-P.: A new multi-channel mac protocol with on-demand channel assignment for multi-hop mobile ad hoc networks. In: ISPAN '00: Proceedings of the 2000 International Symposium on Parallel Architectures, Algorithms and Networks (ISPAN 2000), p. 232. IEEE Computer Society Press, Washington (2000)

8. Lee, U., Midkiff, S.F., Park, J.S.: A proactive routing protocol for multi-channel wireless ad-hoc networks (dsdv-mc). In: International Conference on Information Technology: Coding and Computing (ITCC 2005), vol. 2, pp. 710-715 (2005)

9. Paquereau, L., Helvik, B.E.: A module-based wireless node for ns-2. In: WNS2 2006: Proceeding from the 2006 workshop on ns-2: the IP network simulator, p. 4. ACM Press, New York (2006)

10. network simulator version 2 (ns 2): http://www.isi.edu/nsnam/ns/

11. Perkins, C.E., Bhagwat, P.: Highly dynamic destination-sequenced distance-vector routing (dsdv) for mobile computers. Computer Communications Review 24(4), 234-244 (1994)

12. Katzela, I., Naghshineh, M.: Channel assignment schemes for cellular mobile telecommunication systems: a comprehensive survey. Personal Communications, IEEE [see also IEEE Wireless Communications] 3(3), 10-31 (1996) 